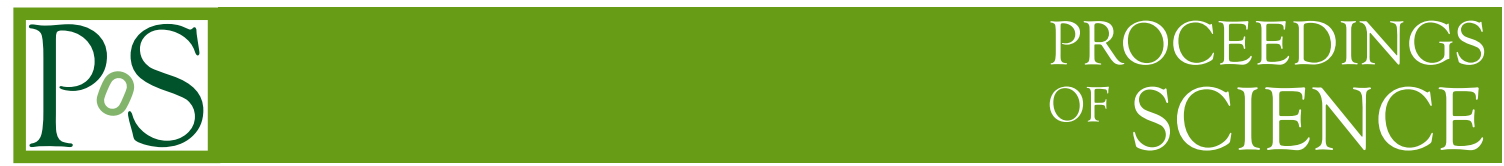

\title{
Beyond Standard Neutrino Theory
}

\section{Toshihiko Ota*}

School of Physical Sciences and Engineering, Yachay Tech, Ecuador

E-mail: totadyachaytech.edu.ed

In this talk, we will discuss phenomenology of new physics associated with neutrinos, in particular, non-standard neutrino interactions, non-unitarity of the lepton mixing matrix, and secret neutrino interactions mediated by a light field.

The 19th International Workshop on Neutrinos from Accelerators-NUFACT2017

25-30 September, 2017

Uppsala University, Uppsala, Sweden

${ }^{*}$ Speaker. 

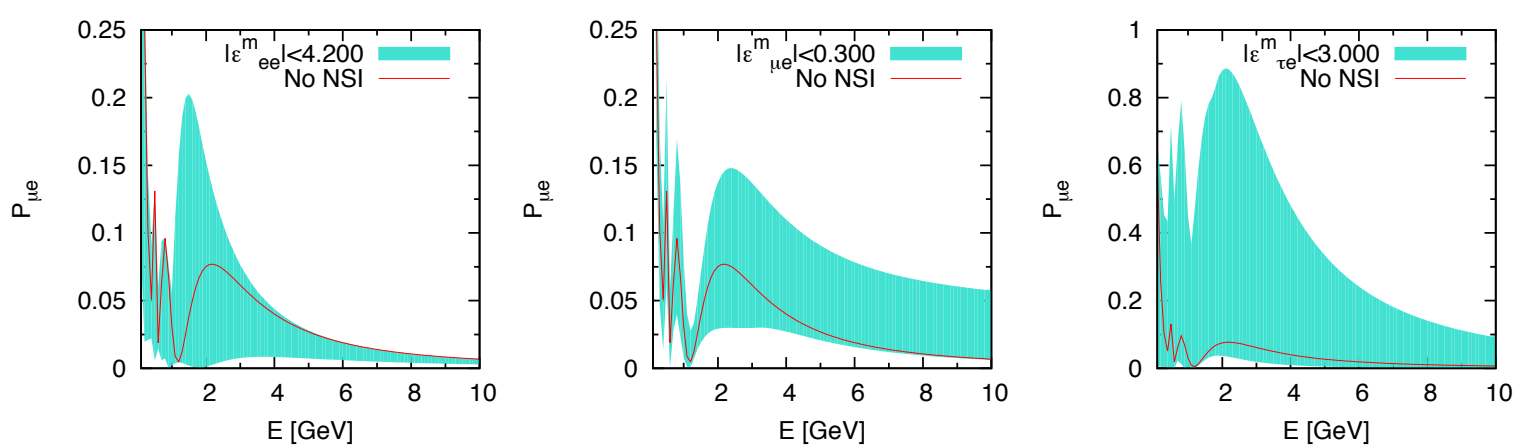

Figure 1: Variations of the oscillation probability for $v_{\mu} \rightarrow v_{e}$ at the DUNE experiment with the change of the NSI parameters in their allowed ranges [2].

\section{Non-standard neutrino interactions}

NSIs are the four-Fermi interactions with neutrino, which are expected to appear in general, if the standard model is an effective theory of fundamental theories realized at the high energy scales, e.g.,

$$
\begin{aligned}
& -\mathscr{L}_{\mathrm{NC}}=2 \sqrt{2} G_{F} \varepsilon_{\alpha \beta}^{m}\left[\bar{v}_{\alpha} \gamma^{\rho} \mathrm{P}_{L} v_{\beta}\right]\left[\bar{e} \gamma_{\rho} \mathrm{P}_{L} e\right]+\text { H.c. }, \Longrightarrow\left(V_{\mathrm{NSI}}\right)_{\alpha \beta}=\sqrt{2} G_{F} N_{e} \varepsilon_{\alpha \beta}^{m}, \\
& -\mathscr{L}_{\mathrm{CC}}=2 \sqrt{2} G_{F} \varepsilon_{\mu \alpha}^{s}\left[\bar{v}_{\alpha} \gamma^{\rho} \mathrm{P}_{L} \mu\right]\left[\bar{d} \gamma_{\rho} \mathrm{P}_{L} u\right]+\text { H.c. }, \Longrightarrow\left|v_{\mu}^{s}\right\rangle=\left|v_{\mu}\right\rangle+\varepsilon_{\mu \alpha}^{s}\left|v_{\alpha}\right\rangle \text { in } \pi^{+} \text {decays. }
\end{aligned}
$$

The neutral current (NC) type NSI with two neutrinos affects the neutrino propagation Hamiltonian as an additional matter effect potential term $V_{\text {NSI }}$. If we have a charged current (CC) type NSI with a neutrino and a charged lepton, it modifies the initial and the final states in neutrino oscillation amplitudes. There is still plenty of room left for them. Particularly, the NC type NSIs are still phenomenologically allowed to be as large as the order of 0.1 or even the order of 1 . For the current upper bounds of the NSI parameters, see e.g., Ref. [1]. As we will see later, it is not so easy to have NSIs with the size of unity in a theoretical point of view. However, we should not exclude the possibilities allowed phenomenologically. In Fig. $\square$ taken from Ref. [2], one can find how much the oscillation probability for $v_{\mu}$ to $v_{e}$ at the DUNE experiment can be modified by the variations of the NSIs in their allowed ranges. Here I would like to overview the phenomenological studies on NSIs, categorizing them into two groups; one is NSIs as noise in the determination of the standard oscillation parameters and the other is NSIs as a signal of new physics.

\subsection{NSIs as noise}

NSIs disturb the determination of the standard oscillation parameters. Here I would like to discuss the impact of the noise produced by the NSIs at the DUNE experiment. The normal hierarchy (NH) case in the standard oscillation scenario is clearly separated from the inverted hierarchy (IH) case at the beam peak (2-3 GeV) of DUNE. However, these two cases start overlapping each other, if NSIs are introduced with the size of the order of $10^{-2}$, as shown in the left plot of Fig. [ [3], and consequently, the sensitivity to the mass hierarchy is reduced. The NSIs also confuse the fit value of the CP violating phase $\delta$. The middle plot in Fig. $\square$ [4] shows that the best-fit value $\delta_{\text {fit }}$ suggested by the DUNE experiment is shifted from the true value $\delta_{\text {true }}$ by the introduction of $\varepsilon_{e e}^{m}$. A serious problem here is, since the fit is not bad (the $\chi^{2}$ is reasonably small), the result looks consistent with the standard oscillation scenario, and therefore, we cannot tell if we have exotic effects or 

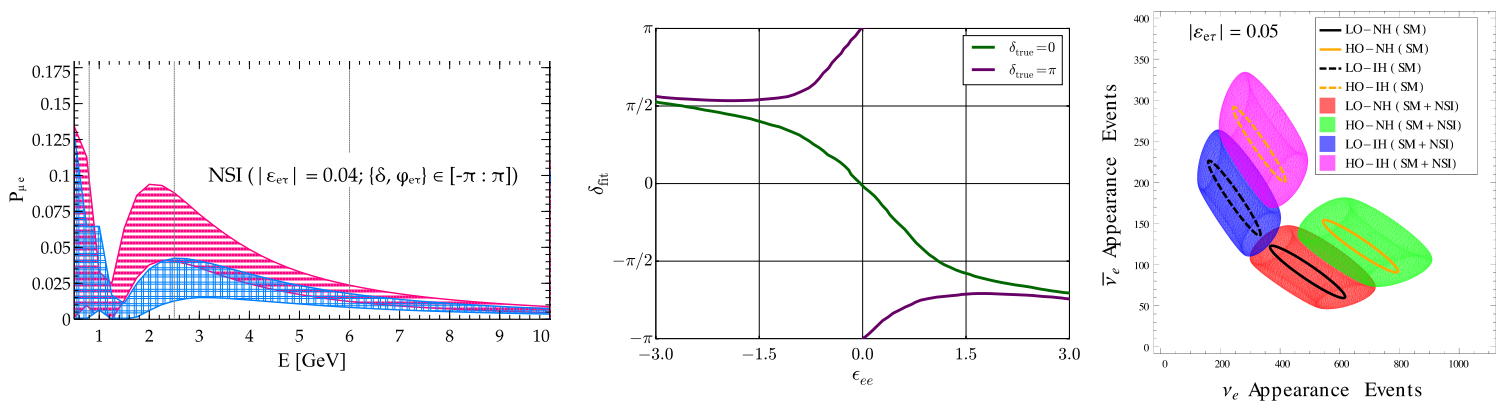

Figure 2: NSIs as noise; NSIs disturb the determination of the standard oscillation parameters at the DUNE experiment. [Left] With $\left|\varepsilon_{e \tau}^{m}\right|=0.04$, the oscillation probability of $\mathrm{NH}$ (red) overlaps with that of $\mathrm{IH}$ (blue) [3]. [Middle] The best-fit value $\delta_{\text {fit }}$ of the $\mathrm{CP}$ violating phase is shifted by the introduction of $\varepsilon_{e e}^{m}$ [4]. [Right] With $\left|\varepsilon_{e \tau}^{m}\right|=0.05$, the ellipses for the lower octant (LO) cases in the bi-probability plane is no more clearly separated from that for the higher octant (HO) cases [5].

not. NSIs also affect the determination of the octant of the 2-3 mixing angle; See the right plot in Fig. 1 [5]. The NSIs can be a serious noise in determining the parameters at the forthcoming long baseline experiments - How can we reduce this noise?

Here I would like to discuss three possibilities. The first thing we can do is to combine the result of $\mathrm{T} 2(\mathrm{H}) \mathrm{K}$ which has shorter baseline than DUNE. Thanks to that, $\mathrm{T} 2(\mathrm{H}) \mathrm{K}$ is less affected by the matter effect and the matter related effects including the NC type NSIs. The authors of Ref. [6] show that the best-fit value of $\delta$ at T2K stays at the true value point even with the $\varepsilon_{e e}^{m}$ with the size of $\sim 1$, although the $\chi^{2}$ for the fit of $\delta$ is damaged a bit. This study suggests that $\mathrm{T} 2(\mathrm{H}) \mathrm{K}$ can determine $\delta$ regardlessly of the existence of the matter NSIs. After the determination of $\delta$ at $\mathrm{T} 2(\mathrm{H}) \mathrm{K}$, the determination of mass hierarchy (and discrimination of non-standard effects) at DUNE becomes easier - DUNE and T2HK are a nice synergetic combination. We can take a step in this direction with a new experiment with a shorter baseline and a good profile beam. As shown in the left plot in Fig. [3 taken from Ref. [7], the fit value of $\delta$ (horizontal axis) at the MOMENT experiment with a baseline of $150 \mathrm{~km}$ does not depend on the value of the NSI $\varepsilon_{e e}^{m}$ at all. There are some plans of muon-based neutrino sources - MOMENT (muon decay in flight) [8], DAEdALUS/IsoDAR (muon decay at rest) [9], and NuSTORM (muon storage ring) [10]. The neutrino beam spectra from those sources are very precisely known, and the intensity of those beams is expected to be high. A high-intensity and relatively low-energy conventional neutrino beam at the European Spallation Source (ESS) is also planed [11]. Such short-baseline, lowenergy, and high-intensity oscillation experiments help reduce the noise from matter NSIs in the determination of the standard oscillation parameters.

The second way to reduce the noise is the use of the results of non-oscillation experiments. In fact, the current experimental bounds to the NSIs are mainly placed by non-oscillation experiments. Recently, the COHERENT experiment has measured the coherent elastic neutrino-nucleon scattering (CEvNS) process for the first time in the history [12], showing that the cross section is consistent with the standard model prediction. The result suggests that the NSI parameters for the Dark solar solution [13] is now excluded at 3 sigma (cf. the right plot in Fig. [1] [14]) if the mass of the field that mediates the NSIs is taken to be heavier than dozens of MeV [15]. This example tells us that the improvement of non-oscillation neutrino experiments is crucially important to exclude 

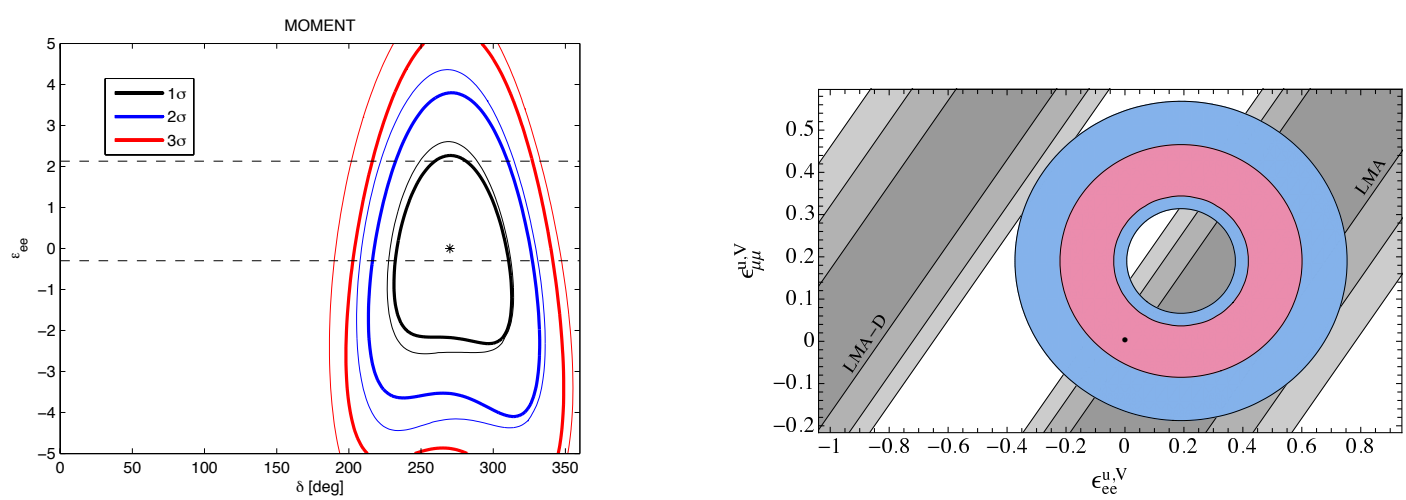

Figure 3: To reduce the noise from NSIs in the determination of the standard oscillation parameters. [Left] Combination with an experiment with a shorter baseline; The MOMENT experiment with the baseline of $150 \mathrm{~km}$ can determine $\delta$ regardlessly of the matter NSIs [7]. [Right] Constraints from non-oscillation experiments; The recent COHERENT result excludes the NSIs for the dark solar solution in the case where the NSIs are mediated by a heavy field [14].

the possible disturbance from large NSIs.

Finally, I would like to remark the importance of tracing out the oscillation curves in a wide range of $L / E$. The distortion in the oscillation curves, which is caused by NSIs, has a characteristic $L / E$ dependence. In the high energy limit with a fixed $L$, all the standard oscillation terms drop quickly as $1 / E^{2}$ or $1 / E^{3}$. The NSI terms of $\varepsilon_{e \mu}^{m}$ in $v_{\mu} \rightarrow v_{e}$ can stay in the high energy limit, because they depend on $1 / E$ and, consequently, it is easy to extract the information of this NSI effect from the oscillation probability at the high energy region. We can separate the NSI effects from the standard oscillation effects and also can separate the different NSI effects by observing the oscillation curves in a wide energy range. Under the standard oscillation assumption, a focused-energy (narrow-band) beam is convenient to determine the oscillation parameters. However, scanning the oscillation probabilities over a wide energy region might be also important to discriminate the non-standard effect and establish the standard oscillation scenario (or discover the exotic effects).

\subsection{NSIs as signal}

There are also many studies on the sensitivities to NSIs at future oscillation experiments Not only at accelerator based experiments but also atmospheric neutrino experiments. Now, the IceCube starts participating in this game [16,17]. At Fig. 团, I would like to introduce a few plot for the sensitivities at DUNE and T2HK, which is taken from Ref. [18]. With the combination of these two experiments, we can explore the NSIs with the size in the range 0.05-0.1. For the sensitivities at the other experiments, see, e.g., Refs. [19,20] (T2HK and atmospheric neutrino at HK), Ref. [1] (INO) and Ref. [21] (ORCA-KM3NeT). The sensitivities to the CC type NSIs at future high intensity facilities are studied in Refs. [22] (MOMENT) and [23] (ESSvSB).

\subsection{Model building for NSIs}

Here I would like to discuss briefly some theoretical aspects of NSIs. Large NSIs such as order of unity are still phenomenologically allowed - Even some of the small tensions in oscillation experiments suggest large NSIs [24-26]. Of course, we should pay attention to the possibilities allowed phenomenologically. However, it is not so easy to accommodate with large NSIs. Naively 

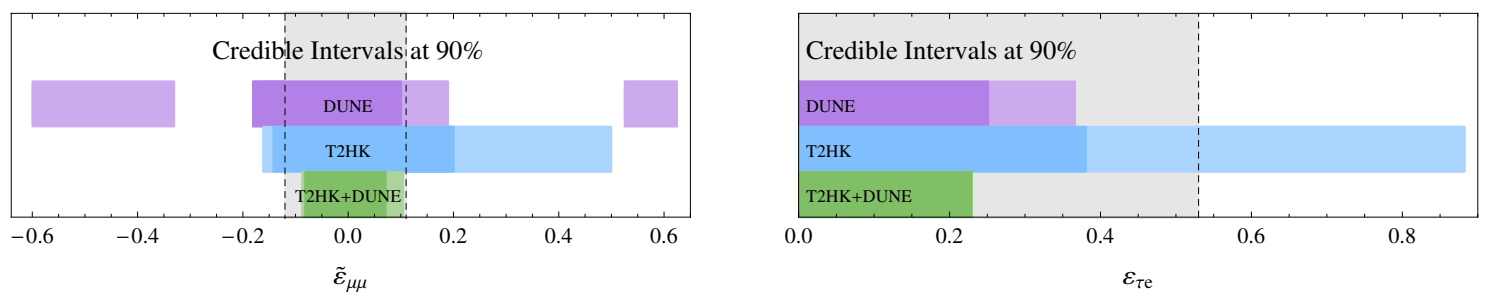

Figure 4: NSIs as signal; Sensitivities of DUNE, T2HK, and their combination to $\tilde{\varepsilon}_{\mu \mu}^{m} \equiv \varepsilon_{\mu \mu}^{m}-\varepsilon_{\tau \tau}^{m}$ and $\varepsilon_{e \tau}^{m}[18]$.

thinking, a mass dimension-six four-Fermi interactions consists of two fundamental interactions mediated by a field such as $Z^{\prime}$, a leptoquark, etc. Setting the couplings of the fundamental interactions to be unity and the mass of the mediator to be TeV, we have the NSI with the size of the order of $10^{-2}$ for the effective coupling $\varepsilon$, which is a kind of a natural size of NSIs we can expect. However, if there is a TeV field with such a large coupling with charged fermions, we should have already found it at the LHC - This naive estimation tells us that the NSIs should be smaller than $\mathscr{O}\left(10^{-2}\right)$. In addition, the NSIs originated from this type of dimension-six operators appear always with the $S U(2)$ counter part with charged leptons. We have to take account of the constraints from the charged lepton counter process. The exhaustive studies on the dimension-six NSIs were already done in 1990s [27,28], and it turned out that the charged lepton processes constrain the NSIs at the level of $10^{-3}$ in general. To avoid the constraints from the charged lepton processes and enjoy the upper bounds of NSIs, the use of dimension-eight operators $(\bar{L} L)(\bar{f} f)\left(H^{\dagger} H\right)$ with four fermions and two Higgs fields was proposed $[29,30]$. The dimension-eight operators do not contain the charged lepton counter part, because the $S U(2)$ on the operators is violated with the Higgs fields. However, they are not free from everything. Here I would like to introduce two arguments on the constraints to the dimension-eight NSIs. First, we have to think about the origin of the effective operators. If the NSI is so large as the order of unity, it should be mediated by tree-level diagrams. The decomposition of the operator to the tree-level diagrams leads to either dimensionsix NSIs $(\bar{v} v)(\bar{f} f)$ or dimension-six non-unitary operators $(\bar{v} \partial \cdot \gamma v)\left(H^{\dagger} H\right)$ or both [31,32]. We can still think of the situation where those dimension-six operators are, somehow, cancelled and only the counter-part-free dimension-eight-origin NSI survives. However, the second argument comes. With the dimension-eight operators such as $(\bar{L} L)(\bar{f} f)\left(H^{\dagger} H\right)$, one can close the outer lines and draw one-loop dimension-six operator $(\bar{L} L)(\bar{f} f)$, and the loop contribution is quadratically divergent $[33,34]$. To regularize this divergence, we need to introduce the dimension-six operator $(\bar{L} L)(\bar{f} f)$ as the counter term. Consequently, we have to have the dimension-six operator in the effective Lagrangian. This means, we are essentially working with dimension-six NSI models. It may not be impossible to solve all these dimension-six mess by adding the terms and tuning the couplings but, as we have seen, it is not so easy to obtain NSIs with the size of their upper bound.

Recently, an alternative way has been discussed to obtain large NSIs, which is NSIs mediated with light mediators. One can obtain the NSIs with the size of $10^{-2}$ with a $\sim 10 \mathrm{MeV}$ mediator with a faint interaction with the couplings of $\sim 10^{-5}$, instead of the $\mathrm{TeV}$ mediator with order-one couplings. A new force mediated by a light field is a kind of trend now, and there are many discussions recently in various contexts, such as cosmic neutrino spectrum, muon anomalous magnetic moment, cosmology (e.g., Refs [35,36]), lepton flavour non-universality in $B$-physics (e.g., Ref. [37]), 


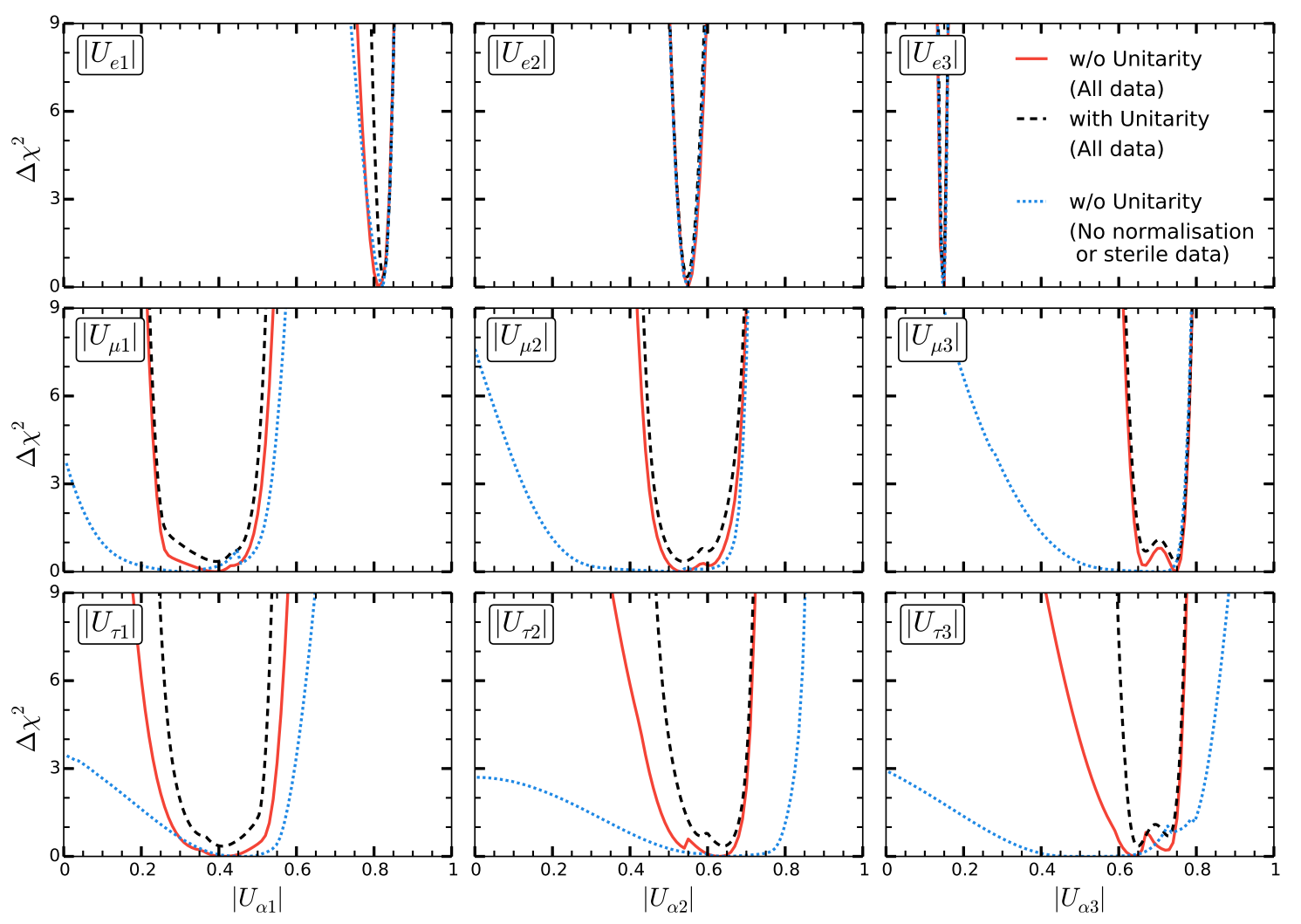

Figure 5: Fit of the elements of the PMNS matrix without the assumption of the unitarity (red) [38].

etc. Neutrino interactions with a light mediator are often labeled as secret neutrino interactions. In the last section, I would like to discuss a scenario of the light leptonic force related to the cosmic neutrino spectrum and muon $g-2$.

\section{Non-unitarity}

Let us turn the topic to the unitarity violation. Although the unitarity is a fundamental feature which is related to the conservation of the probability, the unitarity in the system of active neutrinos can be violated in general. The 3-by-3 PMNS matrix may be a part of the full lepton mixing matrix which is unitary. The extra flavours/generations can participate or not in oscillation, depending on their masses (e.g., Ref. [39]). If the extra generations are light enough to participate in oscillations, we can expect the oscillation signals driven by the extra $\Delta m^{2}$ s. If the extra generations are heavy and cannot participate in the neutrino propagation process, we do not have the extra oscillation signals but the mixing matrix that appears in the oscillation probabilities becomes non-unitary $[40,41]$ - The scenario that neutrinos are mixing with heavy neutral fields is called Minimal Unitarity Violation (MUV) [41]. If neutrinos decay into invisible debris, the Hamiltonian that describes the time evolution of the neutrino system is no more hermitian [42-44]. In such a case, the mixing matrix is not unitary in general.

Our current knowledge on the elements of the PMNS matrix is nicely summarized in Fig. 1 , which is taken from Ref. [38] — The authors fit the elements without assuming the unitarity relations of the PMNS matrix. Since the current oscillation results can be explained with the 3-by-3 
unitary PMNS matrix, the best-fit values of all the elements are consistent with the unitary case. Here you can see that the normalization of the oscillation probabilities set by no-oscillation results in short baseline experiments are important to constrain the elements, in particular, in the $\mu$ and the $\tau$ rows.

In the MUV regime, the non-unitary PMNS matrix $N$ can be parameterized with a hermitian matrix $\eta_{\alpha \beta}(\alpha, \beta \in\{e, \mu, \tau\})$ as

$$
\left(N_{\mathrm{PMNS}}\right)_{\alpha i}=\left(\delta_{\alpha \beta}+\eta_{\alpha \beta}\right) \mathscr{U}_{\beta i}
$$

where $\mathscr{U}$ is the unitary part. ${ }^{1}$ The elements of $\eta_{\alpha \beta}$ are constrained by, among others, the mass of the $W$ boson, the Weinberg angle, muon decay rate, lepton-flavour-violating processes at the level of $10^{-3}$ to 0.05 [48-52]. The non-unitarity $\eta$ modifies oscillation probabilities as a combination of the NSIs [49,53], and the size of the $\varepsilon$ parameters induced by the non-unitarity effect corresponds in general to the order of $\eta$. Therefore, the $\eta$ with the size of 0.05 can be a noise in the parameter determinations at the forthcoming experiments. The constraints to the non-unitarity in the MUV scenario at the future oscillation experiments are discussed in e.g. Refs. [49,54].

\subsection{Unitarity triangle}

We have seen that the current oscillation results are consistent with the unitary PMNS matrix. Is there a way to check the unitarity through the oscillations? - This question was addressed in Refs. [38, 55-57]. In principle, the unitarity of the PMNS matrix can be checked by using one oscillation channel. With the unitary PMNS matrix $U_{\alpha i}$, the oscillation probability in vacuum for $v_{\mu} \rightarrow v_{e}$ consists of the following four terms at the orders of $\left|U_{e 3}\right|^{2}, \Delta m_{21}^{2}\left|U_{e 3}\right|$, and $\left(\Delta m_{21}^{2}\right)^{2}$;

$$
\begin{aligned}
P_{v_{\mu} \rightarrow v_{e}}^{\mathrm{Vac}}= & \overbrace{4\left|U_{\mu 3} U_{e 3}^{*}\right|^{2}}^{\text {coeff. } A} \sin ^{2} \frac{\Delta m_{31}^{2} L}{4 E}+\overbrace{4 \operatorname{Re}\left[U_{\mu 2} U_{e 2}^{*} U_{\mu 3}^{*} U_{e 3}\right]}^{\text {coeff. } B}\left[\frac{\Delta m_{21}^{2} L}{4 E}\right] \sin \frac{\Delta m_{31}^{2} L}{2 E} \\
& -\underbrace{8 \operatorname{Im}\left[U_{\mu 2} U_{e 2}^{*} U_{\mu 3}^{*} U_{e 3}\right]}_{\text {coeff. } C}\left[\frac{\Delta m_{21}^{2} L}{4 E}\right] \sin ^{2} \frac{\Delta m_{31}^{2} L}{4 E}+\underbrace{4\left|U_{\mu 2} U_{e 2}^{*}\right|^{2}}_{\text {coeff. } D}\left[\frac{\Delta m_{21}^{2} L}{4 E}\right]^{2} .
\end{aligned}
$$

Since all the terms have different energy dependencies, one can separate them by tracing out the oscillation probability in a wide energy range and can know the coefficients of these four terms independently. Combining the information of the coefficients $A, D$, (lengths of the sides), and $B$ (the angle between the sides), one can construct a unitarity triangle. The coefficient $C$ is the Jarlskog invariant which is directly proportional to the area of the triangle $[58,59]$. In short, we can check the unitarity by comparing the information brought by $A, B$, and $D$ and the information of $C[55,57]$. This method is spoiled at the high energy region, because the $A$, the $B$, and the $D$ terms behave in the same way as $\propto 1 / E^{2}$ and they cannot be separated any more. Therefore, to carry out this unitarity check, we need to measure the oscillation probability with high precision in the relatively low-energy region. It is tough in the experimental sense, but in principle, we can do it. 

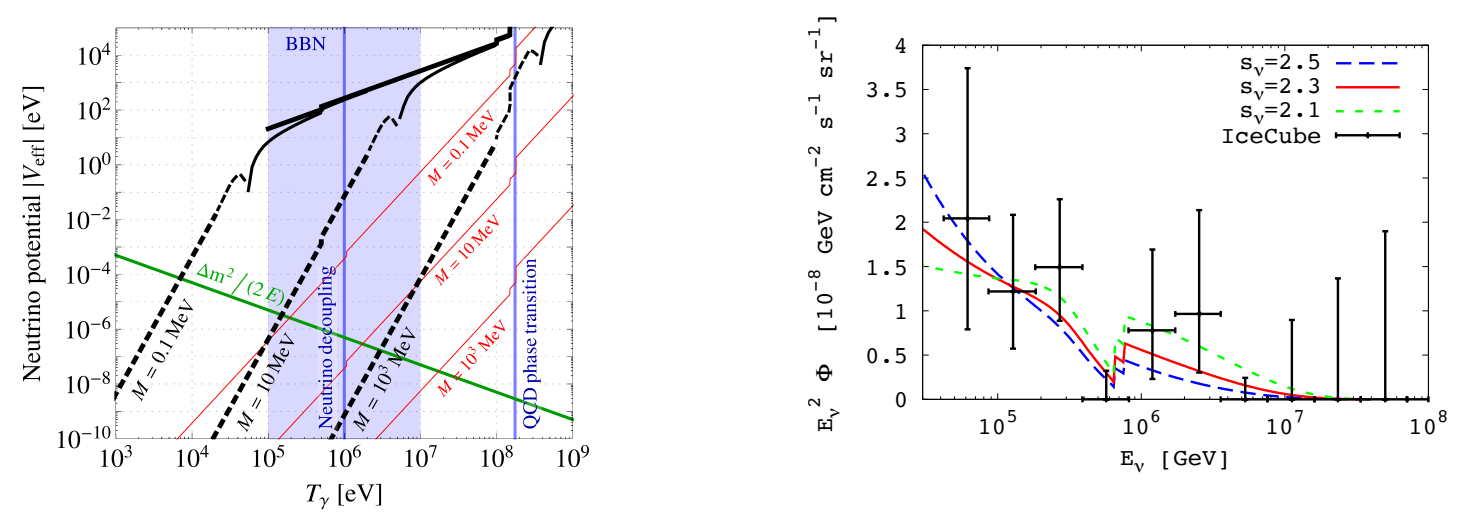

Figure 6: Examples of the studies on secret neutrino interactions. [Left] The interaction between sterile neutrinos, which is mediated by a field with $M \lesssim 10 \mathrm{MeV}$, suppresses the active-sterile mixing during the BBN era [60]. [Right] The $L_{\mu}-L_{\tau}$ interaction mediated by the gauge field with a MeV scale mass can reproduce the gap in the cosmic neutrino spectrum and can simultaneously explain the discrepancy in the muon $g-2$ [61].

\section{Secret neutrino interactions}

Finally I would like to discuss secret neutrino interactions - neutrinos may communicate with the other invisible stuffs or communicate with neutrinos through unknown forces. In a broad sense, NSIs are also in this category, but here we are more interested in the interactions mediated by light fields (lighter than the electroweak scale). They are recently discussed in various contexts. For example, in Refs. $[60,62,63]$ the authors introduced a new gauge force that only sterile neutrino can feel. Thanks to the force, the sterile neutrinos in the early universe get the potential from thermal bath and the active-sterile mixing is suppressed as shown in the left plot in Fig. 6 . This helps the sterile neutrinos to escape the constraint from the effective degree of freedom of the radiation in the era of the Big Bang nucleosynthesis (BBN).

Here I would like to introduce a study on the effect of a secret neutrino-neutrino interaction on the cosmic neutrino spectrum. In 2013, the IceCube collaboration announced the first discovery of the high-energy cosmic neutrino events with the energy of peta electron volts [64]. After the discovery, they have accumulated the events, and now we can know the spectrum of the cosmic neutrinos. Although the statistics are still not enough to conclude the shape of the spectrum, it starts showing some intriguing features (Ref. [65] for 6-year data) - Non-power-law spectrum may be a hint to new physics. A popular idea is that the peaks in the spectrum are made by the decays of the dark matter fields. The other idea is that the gap in the spectrum is made by a resonant scattering. Originally, the spectrum follows a power-law, but the cosmic neutrinos with a particular energy are resonantly scattered with the cosmic neutrino background during their travel to the Earth and lose their energies [61,66-74]. In this scenario, the gap in the spectrum can be understood as the signal of the new neutrino-neutrino interaction. To scatter the sub-PeV cosmic neutrinos resonantly, the new neutrino-neutrino interaction should be mediated by a new field with a mass around the $\mathrm{MeV}$ scale. The coupling of the interaction should be larger than $10^{-4}$ so that the cosmic neutrino can get the scattering at least once during their travels of extra galactic distances $\sim \mathrm{Gpc}$. In the model with the $L_{\mu}-L_{\tau}$ gauge symmetry, the relevant parameter region is still allowed and, in addition,

\footnotetext{
${ }^{1}$ There is another popular way to parameterize the deviation from the unitarity in the MUV [45-48]. For the relation between two parameterizations and the current and future bounds in both the parameterization schemes, see Ref. [49].
} 
the parameter set can also explain the long-standing discrepancy between theory and experiment in the muon anomalous magnetic moment. As shown in the right plot in Fig. 6 , the gap in the cosmic neutrino spectrum is reproduced with the model parameters that can explain the discrepancy in the muon $g-2$.

\section{References}

[1] S. Choubey, A. Ghosh, T. Ohlsson and D. Tiwari, Neutrino Physics with Non-Standard Interactions at INO, DHEP 12 (2015) 126, [1507.0221]].

[2] M. Blennow, S. Choubey, T. Ohlsson, D. Pramanik and S. K. Raut, A combined study of source, detector and matter non-standard neutrino interactions at DUNE, DHEP $\mathbf{0 8}(2016) 090$, [1506.0885]].

[3] M. Masud and P. Mehta, Nonstandard interactions and resolving the ordering of neutrino masses at DUNE and other long baseline experiments, Phys. Rev. D94 (2016) 053007, [11606.05662].

[4] A. de Gouvea and K. J. Kelly, False Signals of CP-Invariance Violation at DUNE, $\square 605.09376$.

[5] S. K. Agarwalla, S. S. Chatterjee and A. Palazzo, Degeneracy between $\theta_{23}$ octant and neutrino non-standard interactions at DUNE, Phys. Lett. B762 (2016) 64-71], [1607.01745]].

[6] S.-F. Ge and A. Yu. Smirnov, Non-standard interactions and the CP phase measurements in neutrino oscillations at low energies, WHEP 10 (2016) 138, [1607.08513].

[7] P. Bakhti and Y. Farzan, CP-Violation and Non-Standard Interactions at the MOMENT, DHEP07 [2016) 109, [1602.07099].

[8] J. Cao et al., Muon-decay medium-baseline neutrino beam facility, Phys. Rev. ST Accel. Beams 17 [2014)090101, [14012.8125].

[9] C. Aberle et al., Whitepaper on the DAEdALUS Program, in Proceedings, 2013 Community Summer Study on the Future of U.S. Particle Physics: Snowmass on the Mississippi (CSS2013): Minneapolis, MN, USA, July 29-August 6, 2013, 2013, ॠ307.2949, https://inspirehep.net/record/1242298/files/arXiv:1307.2949.pdt.

[10] NUSTORM collaboration, D. Adey et al., nuSTORM - Neutrinos from STORed Muons: Proposal to the Fermilab PAC, 1508.6822 .

[11] E. Baussan, M. Dracos, T. Ekelof, E. F. Martinez, H. Ohman and N. Vassilopoulos, The use the a high intensity neutrino beam from the ESS proton linac for measurement of neutrino CP violation and mass hierarchy, 1212.5048.

[12] COHERENT collaboration, D. Akimov et al., Observation of Coherent Elastic Neutrino-Nucleus Scattering, \$cience 357(2017) 1123-1126, [11708.01294]].

[13] O. G. Miranda, M. A. Tortola and J. W. F. Valle, Are solar neutrino oscillations robust?, एसEP 10 (2006) 008, [hep-ph/0406280].

[14] P. Coloma, M. C. Gonzalez-Garcia, M. Maltoni and T. Schwetz, A COHERENT enlightenment of the neutrino Dark Side, 17708.02899.

[15] J. Liao and D. Marfatia, COHERENT constraints on nonstandard neutrino interactions, Phys. Lett. B775(2017) 54-57, [1708.04255].

[16] J. Salvado, O. Mena, S. Palomares-Ruiz and N. Rius, Non-standard interactions with high-energy atmospheric neutrinos at IceCube, [HEP 01 (2017) 14], [1609.03450]. 
[17] ICECUBE collaboration, M. G. Aartsen et al., Search for Nonstandard Neutrino Interactions with IceCube DeepCore, 10709.07079.

[18] P. Coloma, Non-Standard Interactions in propagation at the Deep Underground Neutrino Experiment, DHEP 03 (2016) 016, [1511.06357].

[19] S. Fukasawa and O. Yasuda, The possibility to observe the non-standard interaction by the Hyperkamiokande atmospheric neutrino experiment, Nucl. Phys. B914(2017) 99-116, [1608.05897].

[20] K. J. Kelly, Searches for new physics at the Hyper-Kamiokande experiment, Phys. Rev. D95 (2017)] ए15009, [1703.00448].

[21] KM3NET collaboration, J. A. B. Coelho, Probing new physics with atmospheric neutrinos at KM3NeT-ORCA, in 27th International Conference on Neutrino Physics and Astrophysics (Neutrino 2016) London, United Kingdom, July 4-9, 2016, 2017, 1702.04508, https://inspirehep.net/record/1513471//iles/arXiv:1702.04508.pdt.

[22] J. Tang and Y. Zhang, Study of Non-Standard Charged-Current Interactions at the MOMENT experiment, 1705.09500 .

[23] M. Blennow, S. Choubey, T. Ohlsson and S. K. Raut, Exploring Source and Detector Non-Standard Neutrino Interactions at ESSVSB, DHEP (09 (2015) 096, [11507.02868].

[24] J. Liao, D. Marfatia and K. Whisnant, Nonstandard interactions in solar neutrino oscillations with Hyper-Kamiokande and JUNO, Phys. Lett. B771 (2017) 247-253, [प104.047]]].

[25] J. Liao, D. Marfatia and K. Whisnant, Nonmaximal neutrino mixing at NOvA from nonstandard interactions, Phys. Lett. B767 (2017) 350-353, [1609.01786].

[26] S. Fukasawa, M. Ghosh and O. Yasuda, Is nonstandard interaction a solution to the three neutrino tensions?, 1609.04204

[27] S. Bergmann and Y. Grossman, Can lepton flavor violating interactions explain the LSND results?, Phys. Rev. D59 (1999) 093005, [hep-ph/9809524].

[28] S. Bergmann, Y. Grossman and D. M. Pierce, Can lepton flavor violating interactions explain the atmospheric neutrino problem?, Phys. Rev. D61 (2000) 053005, [hep-ph/9909390].

[29] Z. Berezhiani and A. Rossi, Limits on the nonstandard interactions of neutrinos from e+e-colliders, Phys. Lett. B535 (2002) 207-218, [hep-ph/0111137].

[30] S. Davidson, C. Pena-Garay, N. Rius and A. Santamaria, Present and future bounds on nonstandard neutrino interactions, DHEP (03 (2003) 011, [hep-ph/0302093].

[31] S. Antusch, J. P. Baumann and E. Fernandez-Martinez, Non-Standard Neutrino Interactions with Matter from Physics Beyond the Standard Model, Nucl. Phys. B810 (2009) 369-388, [0807. 1003].

[32] M. B. Gavela, D. Hernandez, T. Ota and W. Winter, Large gauge invariant non-standard neutrino interactions, Phys. Rev. D79 (2009) 013007, [0809.345]].

[33] C. Biggio, M. Blennow and E. Fernandez-Martinez, Loop bounds on non-standard neutrino interactions, DHEP 03 (2009) 139, [0902.0607].

[34] C. Biggio, M. Blennow and E. Fernandez-Martinez, General bounds on non-standard neutrino interactions, DHEP 08 (2009) 090, [0907.009]].

[35] Y. Farzan and S. Hannestad, Neutrinos secretly converting to lighter particles to please both KATRIN and the cosmos, DCAP 1602 (2016) 058, [1510.02201]. 
[36] G.-y. Huang, T. Ohlsson and S. Zhou, Observational Constraints on Secret Neutrino Interactions from Big Bang Nucleosynthesis, 1712.04792.

[37] A. Datta, J. Liao and D. Marfatia, A light $Z^{\prime}$ for the $R_{K}$ puzzle and nonstandard neutrino interactions, Phys. Lett. B768(2017) 265-269, [L102.01099].

[38] S. Parke and M. Ross-Lonergan, Unitarity and the three flavor neutrino mixing matrix, Phys. Rev. D93(2016) 113009, [1508.05095].

[39] C. S. Fong, H. Minakata and H. Nunokawa, A framework for testing leptonic unitarity by neutrino oscillation experiments, $\operatorname{VHEP(02}(2017) 114$, [1609.08623]].

[40] P. Langacker and D. London, Mixing Between Ordinary and Exotic Fermions, Phys. Rev. D38 (1988)] 886.

[41] S. Antusch, C. Biggio, E. Fernandez-Martinez, M. B. Gavela and J. Lopez-Pavon, Unitarity of the Leptonic Mixing Matrix, JHEP10(2006) 084, [hep-ph/0607020].

[42] J. M. Berryman, A. de Gouvea, D. Hernandez and R. L. N. Oliveira, Non-Unitary Neutrino Propagation From Neutrino Decay, Phys. Lett. B742 (2015) 74-79, [1407.663]].

[43] P. Coloma and O. L. G. Peres, Visible neutrino decay at DUNE, 1705.03599.

[44] S. Choubey, S. Goswami and D. Pramanik, A Study of Invisible Neutrino Decay at DUNE and its Effects on $\theta_{23}$ Measurement, 1705.05820 .

[45] F. J. Escrihuela, D. V. Forero, O. G. Miranda, M. Tortola and J. W. F. Valle, On the description of nonunitary neutrino mixing, Phys. Rev. D92 (2015) 053009, [1503.08879].

[46] O. G. Miranda, M. Tortola and J. W. F. Valle, New ambiguity in probing CP violation in neutrino oscillations, Phys. Rev. Lett. 117 (2016) 061804, [1604.05690].

[47] S.-F. Ge, P. Pasquini, M. Tortola and J. W. F. Valle, Measuring the leptonic CP phase in neutrino oscillations with nonunitary mixing, Phys. Rev. D95 (2017) 033005, [1605.01670]].

[48] F. J. Escrihuela, D. V. Forero, O. G. Miranda, M. Tortola and J. W. F. Valle, Probing CP violation with non-unitary mixing in long-baseline neutrino oscillation experiments: DUNE as a case study, Dew Phys. 19(2017)093005, [16/2.0137]].

[49] M. Blennow, P. Coloma, E. Fernandez-Martinez, J. Hernandez-Garcia and J. Lopez-Pavon, Non-Unitarity, sterile neutrinos, and Non-Standard neutrino Interactions, DHEP(04(2017) 153, [1609.08637].

[50] S. Antusch and O. Fischer, Non-unitarity of the leptonic mixing matrix: Present bounds and future sensitivities, DHEP 10(2014) 094, [1407.6607].

[51] S. Antusch and O. Fischer, Probing the nonunitarity of the leptonic mixing matrix at the CEPC, Ent. D Mod. Phys. A31 (2016) 1644006, [1604.00208].

[52] E. Fernandez-Martinez, J. Hernandez-Garcia and J. Lopez-Pavon, Global constraints on heavy neutrino mixing, DHEP 08(2016) 033, [1605.08774].

[53] D. Meloni, T. Ohlsson, W. Winter and H. Zhang, Non-standard interactions versus non-unitary lepton flavor mixing at a neutrino factory, $\operatorname{VHEP(04(2010)041],~[0912.2135].~}$

[54] J. Tang, Y. Zhang and Y.-F. Li, Probing Direct and Indirect Unitarity Violation in Future Accelerator Neutrino Facilities, Phys. Lett. B774 (2017) 217-224], [1] 108.04909]. 
[55] J. Sato, Neutrino oscillation and CP violation, Nucl. Instrum. Meth. A472(2001) 434-439, [hep-ph/0008056].

[56] Y. Farzan and A. Yu. Smirnov, Leptonic unitarity triangle and CP violation, Phys. Rev. D65 (2002) ए300], [hep-ph/0201105].

[57] H. Paes and P. Sicking, Discriminating sterile neutrinos and unitarity violation with CP invariants, Phys. Rev. D95 (2017)075004, [1611.08450].

[58] C. Jarlskog, Commutator of the Quark Mass Matrices in the Standard Electroweak Model and a Measure of Maximal CP Violation, Phys. Rev. Lett. 55 (1985) 1039.

[59] C. Jarlskog, A Basis Independent Formulation of the Connection Between Quark Mass Matrices, CP Violation and Experiment, Z. Phys. C29 (1985) 491-497.

[60] B. Dasgupta and J. Kopp, Cosmologically Safe eV-Scale Sterile Neutrinos and Improved Dark Matter Structure, Phys. Rev. Lett. 112(2014)031803, [11310.6337]].

[61] T. Araki, F. Kaneko, T. Ota, J. Sato and T. Shimomura, MeV scale leptonic force for cosmic neutrino spectrum and muon anomalous magnetic moment, Phys. Rev. D93 (2016)013014, [11508.07471].

[62] X. Chu, B. Dasgupta and J. Kopp, Sterile neutrinos with secret interactions-lasting friendship with cosmology, DCAP 1510 (2015) 011, [11505.02 195]].

[63] J. F. Cherry, A. Friedland and I. M. Shoemaker, Short-baseline neutrino oscillations, Planck, and IceCube, 1605.06506.

[64] IceCube Collaboration collaboration, M. Aartsen et al., First observation of PeV-energy neutrinos with IceCube, Phys.Rev.Lett. $111(2013) 021103$, [1304.5356].

[65] ICECUBE collaboration, J. van Santen, Highlights from IceCube and prospects for IceCube-Gen2 at the 35th International Cosmic Ray Conference (ICRC2017), .

[66] K. Ioka and K. Murase, IceCube PeV-EeV neutrinos and secret interactions of neutrinos, ETEP 2014] (2014)061E0], [11404.2279].

[67] K. C. Y. Ng and J. F. Beacom, Cosmic neutrino cascades from secret neutrino interactions, Phys.Rev. D90(2014) 065035, [11404.2288].

[68] M. Ibe and K. Kaneta, Cv B absorption line in the neutrino spectrum at IceCube, Phys.Rev. D90] (2014) 053011, [1407.2848].

[69] K. Blum, A. Hook and K. Murase, High energy neutrino telescopes as a probe of the neutrino mass mechanism, 1408.3799 .

[70] T. Araki, F. Kaneko, Y. Konishi, T. Ota, J. Sato et al., Cosmic neutrino spectrum and the muon anomalous magnetic moment in the gauged $L_{\mu}-L_{\tau}$ model, Phys.Rev. D91(2015)037301, [1409.4180].

[71] A. Kamada and H.-B. Yu, Coherent Propagation of PeV Neutrinos and the Dip in the Neutrino Spectrum at Ice Cube, Phys. Rev. D92 (2015) 113004, [1504.0071]].

[72] A. DiFranzo and D. Hooper, Searching for MeV-Scale Gauge Bosons with IceCube, Phys. Rev. D92 (2015)095007, [1507.03015].

[73] I. M. Shoemaker and K. Murase, Probing BSM Neutrino Physics with Flavor and Spectral Distortions: Prospects for Future High-Energy Neutrino Telescopes, Phys. Rev. D93 (2016) 085004, [15]2.07228].

[74] Y. Farzan and J. Heeck, Neutrinophilic nonstandard interactions, Phys. Rev. D94(2016) 053010, [1607.07616]. 